CASE REPORT

\title{
Solid pseudopapillary tumour of the pancreas in paediatric and adult age groups
}

\author{
Navami Samaranayake ${ }^{1}$, Akalanka Perera ${ }^{1}$, Sampath Weerakoon ${ }^{1}$ \\ ${ }^{1}$ District General Hospital Monaragala, Monaragala, Sri Lanka
}

\begin{abstract}
Article Information
Total number of

Words 690

Figures 3

Authors have no conflicts of interest to declare

Ethical issues: Informed written consent was taken from the mother in case 1 , and from the patient in case 2 .
\end{abstract}

Keywords: Solid pseudopapillary tumour, pancreas, children, adults

Date of submission: 2020-09-23

Date of acceptance: 2021-04-23

\section{Author responsible for correspondence:}

Navami Samaranayake

House Officer, DGH Monaragala, Monaragala,

Sri Lanka.

Email:navami.sam@yahoo.com

DOI: http://doi.org/10.4038/cjms.v57i1.4976

https://orcid.org/0000-0002-4601-6134

\begin{abstract}
Solid pseudopapillary tumour (SPT) is a rare exocrine tumour of the pancreas predominately seen in females; has a $15 \%$ chance of metastasis; and represents $1-3 \%$ of all pancreatic tumours. We present two case reports of the disease: first a 13 year old female with abdominal pain and vomiting, and second a 36 year old asymptomatic female with an incidental SPT. Both patients had normal biochemical findings with well-defined masses in the pancreas in the contrast enhanced computed tomography. Treatment involved complete resection of the tumour and the latter also had a splenectomy as the tumour was adherent to the splenic vessels. Both remained asymptomatic following surgery and were followed up for a year after resection. We highlight the importance of having a high index of suspicion of the disease entity due to its varied presentation as surgical treatment provides a good prognosis.
\end{abstract}

\section{Background}

Solid pseudopapillary tumour (SPT) is a rare exocrine tumour which represents $1-3 \%$ of all pancreatic tumours [1]. Since described by Frantz in 1959, nearly 3000 cases have been reported, mostly in young females. SPT is identified through imaging while histology with immunohistochemistry give a definitive pathological diagnosis [2]. Treatment involves complete resection of the tumour [3]. We present two different presentations of SPT in paediatric and adult age group.

\section{Case 1}

A previously healthy 13 year old female presented with colicky central abdominal pain of one day duration, which migrated to the epigastrium. Pain radiated to the back; persisted when bending forward; and had vomiting and chest tightness without precipitators. Her examination was unremarkable. After getting treated for gastroesophageal reflux disease, she was admitted again; thus, was managed as acute pancreatitis. Despite normal biochemical investigations including serum amylase, her complains remained. 
Her abdominal ultrasound scan revealed an enlarged pancreas with a solid cystic lesion. contrast enhanced computed tomography (CECT) of her abdomen revealed a definite, hypo-attenuating cystic mass in the mid body of the pancreas with suspicious solid component more in favour of SPT (Figure 1). She underwent a distal pancreatectomy and during surgery, there were no signs of metastasis or abdominal free fluid. Histological examination confirmed the diagnosis of a SPT (Figure 2).

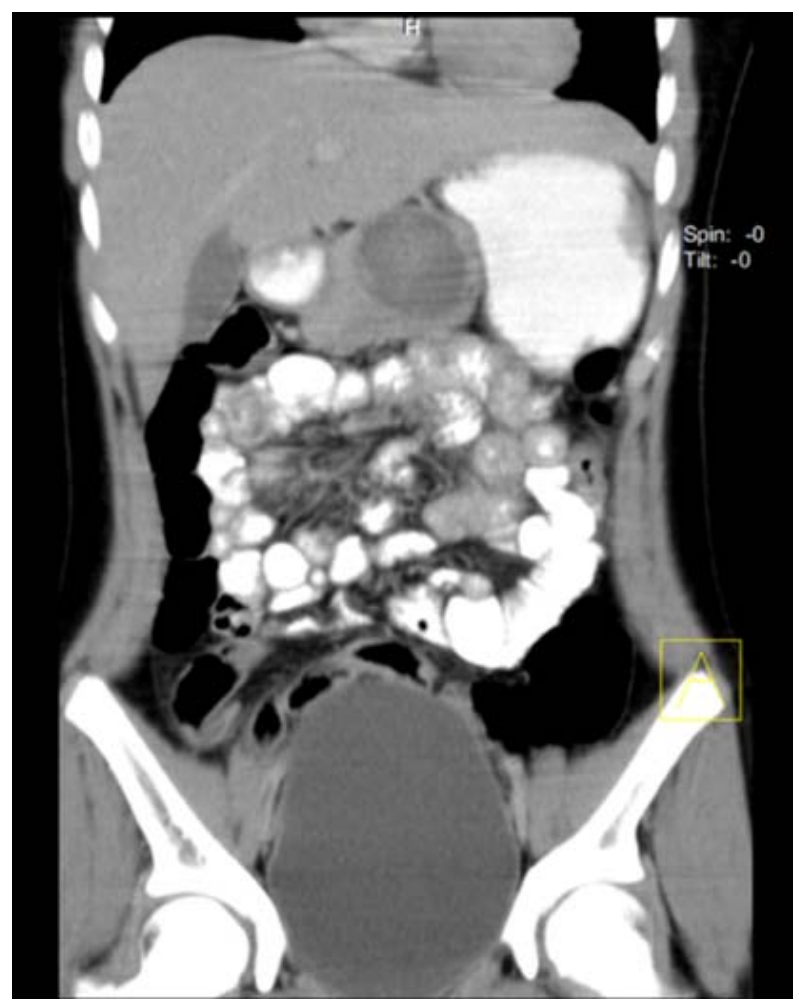

Figure 1. CT venous image of the SPT of the pancreas of the 13 year old female.

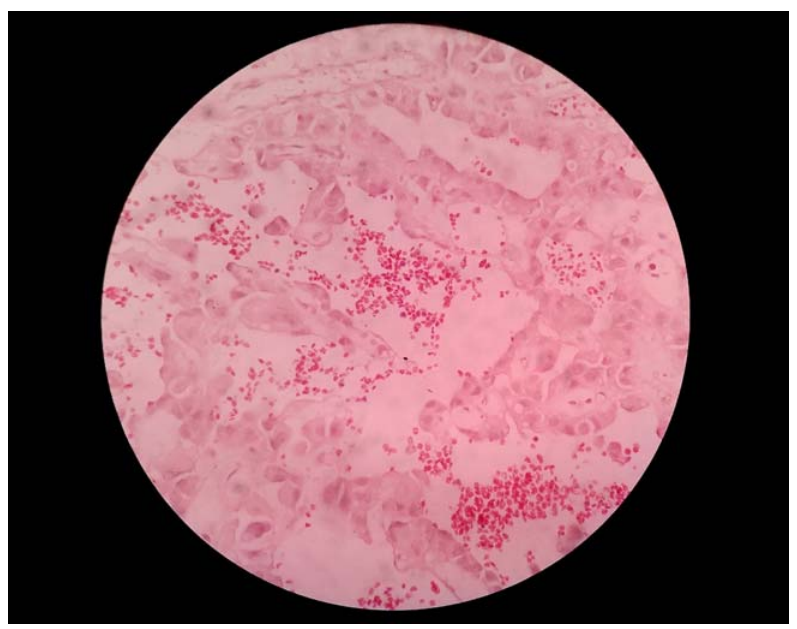

Figure 2. Histological slide of pancreas of the 13 year old patient with SPT.

\section{Case 2}

A 36 year old sub-fertile female presented with isolated left sided lower abdominal pain for 6 months. Her clinical and biochemical findings were unremarkable. The transvaginal scan showed a left ovarian cyst and she underwent laparoscopic bilateral ovarian cystectomy. The histology report depicted bilateral ovarian endometriomas. During investigations, a non-contrast CT showed a pancreatic tail lesion. The CECT showed a $5 \mathrm{~cm}$ defined solid-cystic lesion in the pancreatic tail without local invasion or metastasis, likely mucinous cystic pancreatic neoplasm (Figure 3). During distal pancreatectomy, splenectomy was also performed as the splenic vein was tightly adhered to the tumour. Histology of the specimen showed features of SPT without infiltrations to the pancreas or perinephric fatty tissue; and no neurovascular invasions or lymph nodes. Spleen was unremarkable. The patient was vaccinated against encapsulated organisms and was started on oral penicillin.

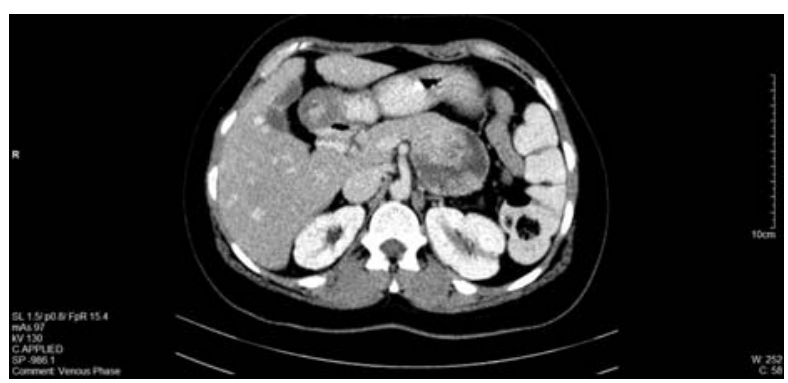

Figure 3. CT image of the SPT of the pancreas of the 36 year old female.

\section{Discussion}

SPT, otherwise known as Frantz tumour results in 1-3\% of all pancreatic tumours [1]. Though infrequent in males or children, they include the majority (70\%) of paediatric pancreatic neoplasms [3, 4]. Its female preponderance has been hypothesized to be, because of the juxtaposition of the primordial pancreatic cells to the ovarian ridge in the embryonic phase [5]. This slow growing exocrine tumour with a low malignant potential, has a mean age of presentation of 22 years [1].

As in the case 2, most SPTs are incidentally identified. They can also present with abdominal pain, vomiting, and other nonspecific manifestations as evident in case 1 $[2,6,7]$. Unlike other pancreatic tumours features such as jaundice, weight reduction and pancreatitis are rare in SPT [7]. These were unnoticeable in the reference cases. Imaging techniques such as ultrasound and CT scan recognize the heterogeneous masses with solid and cystic components [2]. Endoscopic fine needle aspiration (FNA) may diagnose when uncharacteristic imaging results are found pre-operatively [1, 2]. Although imaging is adequate for surgical diagnosis, the ultimate confirmation relies on 
histology and immunohistochemistry [2]. Macroscopically, SPT is well circumscribed with solid and cystic regions [8]. Microscopically, they have a distinctive appearance of solid regions coupled with regions of pseudopapillae surrounding thin blood vessels [8]. Neurovascular invasion is uncommon, if present it indicates aggressiveness [8]. Our patients lacked neurovascular invasion and remained asymptomatic even one year after surgery.

In SPT $\alpha 1$-antitrypsin, CD56, CD10, and vimentin remain elevated [1, 2]. Metastasis is seen up to $15 \%$ of cases, mostly restricted to the liver or peritoneum [1] without lymphatic spread [7]. Its overall 5 year survival rate is $97 \%$ after surgical resection [1]. Even with liver metastasis or local recurrence, treatment remains the same [3].

\section{Learning points}

SPT is rare; has a low malignant potential; with a long term survival rate of $97 \%$ with timely surgical intervention. It is usually incidentally diagnosed in females through imaging. Even in children, diseases that mimic pancreatitis need to be evaluated.

\section{References}

1. Shuja A, Alkimawi KA. Solid pseudopapillary tumor: A rare neoplasm of the pancreas. Gastroenterol Rep (Oxf). 2014 May; 2(2): 145-9. doi:10.1093/gastro/gou006
2. Cai Y, Ran X, Xie S, et al. Surgical management and longterm follow-up of solid pseudopapillary tumor of pancreas: A large series from a single institution. J Gastrointest Surg. 2014 May; 18(5): 935-40. doi:10.1007/s11605-014-2476-6.

3. Casamassima MGS, Gause CD, Goldstein SD, et al. Pancreatic surgery for tumors in children and adolescents. Pediatr Surg Int. 2016; 32(8): 779-788. doi:10.1007/s00383-016-3925-y

4. Vassos, N, Agaimy A, Klein P, et al. 2015. Solidpseudopapillary neoplasm (SPN) of the pancreas: An enigmatic entity. Int J Clin Exp Pathol. 2013; 6(6): 1051-9.

5. Kosmahl M, Seada LS, Jänig U, et al. Solid-pseudopapillary tumor of the pancreas: Its origin revisited. Virchows Arch. 2000 May 12; 436(5): 473-80.

6. Lubezky N, Papoulas M, Lessing Y, et al. Solid pseudopapillary neoplasm of the pancreas: Management and long-term outcome. Eur J Surg Oncol. 2017 Jun; 43(6): 1056-60. doi: 10.1016/j.ejso.2017.02.001.

7. Law JK, Ahmed A, Singh VK, et al. A systematic review of solid-pseudopapillary neoplasms: Are these rare lesions? Pancreas. 2014 Apr; 43(3): 331-7. doi:10.1097/MPA.0000000000000061.

8. Laje P, Bhatti TR, Adzick NS. Solid pseudopapillary neoplasm of the pancreas in children: A 15-year experience and the identification of a unique immunohistochemical marker. J Pediatr Surg [Internet]. 2013 Oct; 48(10): 2054-60. doi:10.1016/j.jpedsurg.2013.02.068. 